AperTO - Archivio Istituzionale Open Access dell'Università di Torino

\title{
Assessing the Personality Profile with ADHD Characteristics Using the Rorschach Performance Assessment System (R-PAS)
}

\section{This is the author's manuscript}

Original Citation:

\section{Availability:}

This version is available http://hdl.handle.net/2318/1703939

since 2019-06-04T18:27:08Z

Published version:

DOI:10.1007/s10826-019-01359-y

Terms of use:

Open Access

Anyone can freely access the full text of works made available as "Open Access". Works made available under a Creative Commons license can be used according to the terms and conditions of said license. Use of all other works requires consent of the right holder (author or publisher) if not exempted from copyright protection by the applicable law. 
ADHD AND THE RORSCHACH

\begin{abstract}
Assessment of ADHD typically includes rating scales completed by parents, teachers, and/or patients themselves. However, rating scales may be subject to rater bias effects, and raters may not recognize the patient's implicit qualities and underlying personality processes. In contrast, the Rorschach test permits standardized, in vivo observation and coding of behaviors as outcomes of implicit personality processes, and, thus, it may assist clinicians in the formulation of contextualized treatment decisions. By using the Rorschach Performance Assessment System (R-PAS), a performance-based personality test characterized by empirical foundation and psychometric efficiency, we conducted an observational study to investigate in depth personality and its implicit qualities in children with ADHD who were not on medication. After reviewing thoroughly the previous studies on ADHD and the Rorschach and discussing their contradictory findings, we compared data of our sample $(N=31)$ with the R-PAS normative children's group. Several Rorschach variables differed from RPAS norms and indicated the presence of unconventional perceptions characterized by non-cognitively mediated interactions and difficulties mentalizing human behavior. Overall, it appears that hyperactivity and impulsivity are associated with unconventional and sometimes mistaken processing of information especially relevant to people and relationships. Although we compared the Rorschach profiles to norms rather than a matched control group, our findings could provide useful information for understanding the personality functioning beyond manifest symptoms or symptom reports of children with ADHD.
\end{abstract}

Keywords: Rorschach; R-PAS; ADHD; personality functioning; unconventional perception. 
ADHD AND THE RORSCHACH

\section{Assessing the personality profile with ADHD characteristics using the Rorschach Performance}

\section{Assessment System (R-PAS)}

Attention-Deficit/Hyperactivity Disorder (ADHD) is among the most commonly diagnosed disorders of childhood, yet it fuels a broad and controversial debate in the scientific community (Barkley, 2015; Wilens, Biederman, \& Spencer, 2002). In the Diagnostic and Statistical Manual of Mental Disorders, Fifth Edition (DSM-5 American Psychiatric Association, 2013), ADHD is listed in the new category of Neurodevelopmental Disorders, which includes a group of conditions with onset during the developmental period associated with specific impairments in executive functions (i.e., inability to plan, focus attention, remember instructions, and juggle multiple tasks successfully; Barkeley, 1997, 2000; Doyle, 2006; Vaidya, 2012). Indeed, in ADHD, a persistent pattern of inattention (e.g., poor concentration and difficulties maintaining focus on tasks) and/or hyperactivity/impulsivity (e.g., making restless movements, excessive talking, and difficulties awaiting turns, taking risks) interferes with the overall functioning and development.

Assessment of ADHD typically includes rating scales [e.g., Behavior Assessment System for Children (BASC; Reynolds \& Kamphaus, 1992); Conners’ Parent Rating Scales -Revised; (CPRS-R; Conners, Sitarenios, Parker, \& Epstein, 1998)] that are completed by parents, teachers, and/or patients themselves. Although these rating scales may be subjected to rater bias effects (Martel, Schimmack, Nikolas, \& Nigg, 2015), parents and teachers are considered the most valid sources of information in the evaluation of ADHD. Presumably the structured and social context with readily available comparisons to similarly aged children reduces error in teachers' ratings.

The Rorschach test permits standardized, in vivo observation and coding of behaviors that may reflect implicit qualities and underlying personality processes not recognized by parents or teachers (Viglione, 1999). In addition, the ADHD is embedded in a wide variety of psychological, behavioral, and systemic contexts; a multi-purpose performance-based personality and processing test, like the Rorschach, could contextualize and add meaningful information to parent and teacher rating scales. Therefore, it might provide additional, incremental information that may assist clinicians in the formulation of contextualized treatment decisions.

The Rorschach test, itself, is a problem-solving task based on perception that provides standardized behavioral observations of how examinees make visual attributions to the stimuli, provide verbal and nonverbal communications about them, and interact with the examiner, the inkblot, and the external assessment setting (Meyer, 2017). More specifically, the Rorschach test consists of 10 ambiguous but evocative, inkblot designs. 
ADHD AND THE RORSCHACH

Respondents are asked to communicate to the examiner what they see in the inkblots. Subsequently, their responses are then interpreted based on (a) what they see, (b) what, in the inkblot, made them see what they saw (e.g., the shape, the color, etc.), and (c) where in the inkblot they were looking at. By comparing the examinee’s scores to norms, the examiner can infer both internal (e.g., intrapsychic processes, thoughts, impulses, and feelings) and external (e.g., social relations) experiences. For example, content themes and indicators of confused or logical thinking are thought to represent real-life behavior and cognitive processing.

Despite the potential contribution of the Rorschach to understanding the ADHD syndrome, only a few previous studies used the Rorschach test to study ADHD and its correlates (e.g., Bartell \& Solanto, 1995; Cotugno, 1995; Meehan et al., 2008). In most of these studies, the Rorschach was administered and scored according to the Comprehensive System (CS; Exner, 1974, 2003). After a brief introduction to the CS, these ADHD/Rorschach studies are reviewed in chronological order.

The CS was developed to create an integrated system including the five dominant systems used in the U.S. at that time (Beck, Hertz, Klopfer, Piotrowski and Rapaport). The aim was to provide a psychometric foundation for the scoring and interpretation of the test, so to meet validity and reliability standards for research and clinical practice. A recent publication incorporating many meta-analyses (Mihura et al., 2013) identified numerous strongly supported Rorschach scores, increasing the knowledge on the validity of the Rorschach test in both research and clinical settings.

The first study on personality features in children with ADHD using the Rorschach test was conducted by Gordon and Oshman in 1981. This study’s investigators administered and coded the Rorschach according to Klopfer et al. (1954), a predecessor of the CS. By comparing the Rorschach protocols of 20 male children characterized as hyperactive (ages 6 to 11) with 20 non-hyperactive male children, the authors found that children with hyperactivity produced fewer Human Movement (M) and Human Content (H) responses and more Animal Content (A) responses than the non-hyperactive group. Fewer $\mathrm{M}$ and $\mathrm{H}$ responses suggest a less favorable understanding of people and relationships, whereas high frequencies of animal responses may indicate cognitive simplicity, immaturity or evasiveness (Exner, 2003; Meyer et al., 2011). Furthermore, age-related effects occurred within the hyperactive group, so that younger hyperactive children provided lower H\% (percentages with Rorschach variables are typically the proportion of the responses $(\mathrm{R})$ in a given protocol with the named variable, that is $\mathrm{H} / \mathrm{R}$; for example, $\mathrm{H} \%$ in this case would refer to the total percent of responses with human content), more distorted form (X-\%) and color (SumC) responses, and shorter reaction times than the older hyperactive children, suggesting a plausible decrease in impulsivity with increasing age in the hyperactive 
ADHD AND THE RORSCHACH

group. According to the Rorschach literature, color responses are thought to be related to high reactivity to environmental stimuli, impulsivity, and, at worst, uncontrolled emotional reactions, whereas X-\% suggests atypical perceptions (Klopfer et al., 1954; Meyer et al., 2011; Mihura et al., 2013). Taken together, these ADHD findings suggest immature processing, defensiveness, and the experience of difficulties managing in the world because of misinterpretations of situations.

Of course, Gordon and Oshman’s (1981) exploratory study has some limitations (Bartell \& Solanto, 1995). First, the children were classified as hyperactive only by using behavior-rating scales, and no other tool or algorithm was used to confirm the ADHD diagnosis. Second, children in both the hyperactive and the control groups were diagnosed with other psychiatric disorders that could have influenced the children’s performance during the Rorschach administration.

Subsequently, Bartell and Solanto (1995) compared 24 Rorschach protocols of children (ages 5 to 11) that met DSM-III-R criteria of ADHD with age-based norms (Exner, 1993). The authors hypothesized that impulsivity, poor attention span, and increased reactivity to external stimuli would be associated with color dominant responses (CF), a lower Form Dominance Proportion (FC:CF+C), fewer M's, lower scores on Experience Actual [EA; sum of M and the weighted sum of Color responses (SumM:WsumC)], greater X-\% scores, and the same number of common, easily seen details (D) when compared to the norms. Their results supported only the hypotheses related to $\mathrm{M}, \mathrm{X}-\%, \mathrm{D}$, and EA. The sample with ADHD, indeed, showed limited empathic ability (M), difficulty in translating perceptual inputs appropriately, and poor reality testing (X-\%), presumably because they provided the responses impulsively. D did not differ significantly from the norms, thus indicating that the children with ADHD did not have poor abilities to perceive the more obvious characteristics of the inkblots (Exner, 1993).

Bartell and Solanto (1995) also investigated the differences in Rorschach protocols between two sample subgroups: one with ADHD and Oppositional Defiant Disorder (ODD), the other with ADHD but without ODD. They hypothesized that children with both ADHD and ODD would have elevated aggressive scores (AG) and lower frequencies of H responses, compared to children with ADHD only, as an expression of limited empathy and also hostile views of other people (Exner, 1993, 2003). The ODD subgroup gave fewer H responses, but the frequency of AG responses was the same in the two subgroups. One possible explanation for these findings was that the Rorschach indices of aggressiveness might be correlated only with disorders characterized by severe acting-out behaviors (i.e., Conduct Disorder). Given that the ADHD/ODD group had significantly fewer $\mathrm{H}$ contents than the normative group and that no differences were found for the number of 
ADHD AND THE RORSCHACH

AG responses, the authors concluded that the number of $\mathrm{H}$ responses characterized the ODD subgroup better than the number of AG responses.

Cotugno (1995) compared Rorschach protocols of 120 children (ages 5 to 6) with ADHD with both non-ADHD clinical $(N=40)$ and non-clinical $(N=40)$ control groups. The author hypothesized that children with ADHD would oversimplify the stimulus field (e.g., High Lambda) and perceive reality as more distorted (e.g., X-\%) when compared to the control groups. Lambda, which is calculated as the ratio of the simple pure form responses divided by more complex and elaborated non-pure form responses, is considered to measure the extent to which a person is closed or open to internal and external experience, ambiguity, and complex situations (Weiner, 1998). A significantly higher score was obtained by the ADHD sample as compared to both the clinical and non-clinical samples on the Coping Deficit Index (CDI), suggesting that the children with ADHD experienced a deficit in adaptive resources and in interpersonal relationships. High scores on this variable has also been demonstrated to be related to simplicity and low productivity overall in a record (Meyer et al., 2011). Similarly, as would be expected, Cotugno (1995) also found that the sample with ADHD had a significantly higher Lambda than the non-clinical and clinical samples. The ADHD sample also showed higher scores on the Sum of Shading (SumSh) and the Depression Index (DEPI), suggesting painful affect and depressive features, and lower Affective Ratio (Afr), [(3r+(2)/R], Pure H, Popular $(\mathrm{P})$ responses, and good form quality $(\mathrm{X}+\%)$, than the non-clinical control group. Overall, the results could be interpreted to suggest that ADHD is related to more intense features of isolation and discomfort, less social involvement, avoidance of decision-making, and dependence, while the clinical control group demonstrated a significantly higher level of perceptual and thinking mistakes [Schizophrenia Index (SCZI), Weighted Sum Form of the first six Special Scores (WSum6), X-\%, and Unusual Form quality (Xu\%)].

Jain, Sing, Mohanty, and Kumar (2005) recruited 224 Indian children (ages 6 to 11) divided into two groups: children with ADHD $(N=111)$ and non-clinical controls $(N=113)$. The Rorschach Inkblot Test (RIT; Beck \& Molish, 1967) and the Somatic Inkblot Series (SIS-I; Cassell, 1980) were individually administered to each participant. Several Rorschach indices showed significant differences between the two groups: Children with ADHD produced lower conventional pure form (F+\%), M, D, P (Popular) responses, and animal content (A\%) than the non-clinical, control group. Lower $\mathrm{M}, \mathrm{F}+\%$, and $\mathrm{A} \%$ in the ADHD group may indicate, respectively, lack of capacity for using inner resources for coping and poor empathy (Exner, 2003; Klopfer et al., 1954), distractibility, poor concentration, and unconventional or peculiar perceptions of the world (Beck et 
ADHD AND THE RORSCHACH

al.,1961; Meyer et al., 2011). Color responses (i.e., C, CF, FC) were higher in the ADHD group than in the control group, indicating reactivity, and possibly impulsivity and emotional excitability.

Zhong, Jing, Wang, and Yin (2007) studied the Rorschach recruiting 40 children with ADHD and 40 healthy, control participants. The ADHD group showed significantly higher scores in AG, Lambda, SumSh, X\%, SCZI, DEPI, and CDI, and lower scores in organizational Activity (Zf), Z, COP, Egocentricity Index, and $\mathrm{X}+\%$ than the control group. Therefore, the ADHD group was characterized by emotional reactivity, depressive features, impaired cognitive functions, poor self-consciousness and interpersonal interactions, and aggressive behavior.

Finally, Meehan and colleagues (2008) focused on emotional self-regulation and internal resources and ADHD using the Rorschach Inkblot Method (RIM; Exner, 1993; Rapaport, Gill, Schafer, \& Holt, 1968); in this study the CS administration was adopted as well as in the aforementioned studies, but with the exception that the inquiry followed each spontaneous response, as has been recommended for young children (Ames, Metraux, Rodell, \& Walker, 1974). ADHD inclusion criteria was the presence of at least six of the nine DSM-IV TR diagnostic criteria from either the inattentive or hyperactive categories, and a clinically significant score on the DSM-IV ADHD rating scale (DuPaul et al., 1997) completed by parents, teachers, and/or clinicians. The authors hypothesized that Rorschach variables would be associated with specific impairments in ADHD: vulnerabilities (e.g., few color contents), overall scarce capacity to access internal resources (lower EA score), oversimplified and distorted interpretation of reality (higher Lambda, lower D and X+\%), and poor representations of social interactions, (e.g., fewer M’s, as well as fewer depictions of human or human-like figures in general [H, Hd, (H), (Hd)]). Comparisons between the Rorschach responses of the ADHD group $(N=28)$ with those of a nonspecific clinical group $(N=14)$ revealed no significant differences in $\mathrm{H}$ contents and $\mathrm{X}+\%$, but the ADHD group did have significantly lower scores on the EA, a measure of internal coping resources. Finally, children with ADHD gave significantly fewer $\mathrm{M}$ responses than the comparison group. These findings were regarded as being "consistent with the notion that children with ADHD may have difficulty accessing internal resources in the face of high stimulus demand in order to organize, process, and represent their experience” (Meehan et al., 2008, p. 452).

Integrating the Rorschach research findings, key features in ADHD seem to be related to cognitive simplicity (L, X-\%, X+ \%, F-\%, Z scores), problems in social interactions and relationships (H, COP, M), and inadequate inner resources for coping and inaccurate perception of the world (SumSh, CDI, X-\%, EA, P). Furthermore, children with ADHD suffer from distressing or irritating internal stimuli often with depressive 
ADHD AND THE RORSCHACH

features (Sum Sh, DEPI, Afr). Some of the findings are in line with the most recent meta-analysis of the Rorschach using the CS approach (Mihura et al., 2013) concerning limited empathy, overall insufficient mental abilities including planning, imagination (M), and poor cognitive and emotional resources (EA) as significantly related to ADHD, as well as the presence of frustration (AG) and impulsivity or reactivity (i.e., $\mathrm{CF}+\mathrm{C}>\mathrm{FC}$; Pure C) as related to the externalizing behavior problems (Mihura et al., 2013).

Previous studies on Rorschach and ADHD provided incomplete information about the possible interaction between ADHD and gender. Indeed, the scientific literature on the ADHD indicates that girls with ADHD showed significantly lower levels of hyperactivity, inattention, impulsivity, externalizing problems, and higher levels of internalizing problems compared to males with ADHD (e.g., Gershon, 2002). Furthermore, the Rorschach and ADHD studies produced contradictory findings: Indeed some of the previous studies reported the presence of immature and elementary emotional representations (D) with impulsivity and restless, either expressed or experienced, while others identify features associated with a responsiveness primarily to sensory but not affective cues, as well as low reactivity in children with ADHD (A, C, CF, FC, Sum C, AG.). Moreover, accumulated research suggests that using the CS norms makes children and adolescents, as well as adults, look excessively pathological (Hamel \& Shaffer, 2007). In other words, relying on CS norms vs. Composite International Reference Values (CIRV; Meyer, Erdberg, \& Shaffer, 2007) would suggest different inferences on form quality, color, and human representation classes of variables and would result in interpretations that are more pathological in terms of (a) perception and thinking, (b) cognitive and emotional features, and (c) representations of human relationships and social interactions (Viglione \& Giromini, 2016). Therefore, the typical or average child or adolescent in the international sample would be considered to have key pathological or problematic personality features if CS norms are used.

The current study aimed to evaluate personality and processing features associated with persistent patterns of inattention and/or hyperactivity-impulsivity in children with ADHD in the absence of medication. By using the Rorschach Performance Assessment System (R-PAS; Meyer et al., 2011), we explored personality and processing psychological functions in children with ADHD.

The aim of R-PAS is to enhance psychometric and international foundations of Rorschach assessment. First, R-PAS administration includes procedures aimed at constraining the number of responses per protocol, to improve the psychometric efficiency of the test, and reduce the number of overly short, poorly informative records (Reese, Viglione, \& Giromini; 2014). Second, some CS variables are not included in R-PAS, others are included but with some variations (Viglione, Giromini, Gustafson, \& Meyer, 2014), and a few other variables 
ADHD AND THE RORSCHACH

that were not part of CS are included in R-PAS. Third, differently from CS, R-PAS draws on internationallybased (rather than U.S.) normative reference data, consistent with emerging research indicating that CS norms notably differ from many nonclinical samples from all over the world (Giromini, Viglione, \& McCullaugh, 2015). Finally, recent studies provided further evidence on the reliability (Kivisalu, Lewey, Shaffer, \& Canfield, 2016; Lewey, Kivisalu, \& Giromini, 2018; Pignolo et al., 2017) and validity (e.g., Ando’ et al. 2015; Giromini et al., 2016) of R-PAS scores. Thus, R-PAS may represent an innovative way of measuring behavioral problems and their correlates in children and adolescents with ADHD.

\section{Method}

\section{Participants}

We used archival data from an outpatient, neuropsychiatric agency at the hospital of Cuneo, Italy. We considered 54 children with a primary diagnosis of ADHD by expert clinicians, based on the clinical criteria outlined in the DSM-IV-TR. Inclusion criteria were: (a) a primary diagnosis of ADHD; (b) a t-score of 64 or greater in at least one of the two Conners’ Parent ADHD DSM scales (CPRS-R; Conners, 1998) completed by both the mother and the father of each child; and (c) the children were not on medication when the Rorschach was administered. The final sample was composed of 31 Caucasian children ( 25 boys and 6 girls), ranging in age from 7 to 17 years $(M=11.90 ; S D=2.71)$ having one to ten years of education $(M=6.06 ; S D=2.52)$.

\section{Procedure}

The outpatient, neuropsychiatric agency at the hospital of Cuneo, Italy, is specialized in the treatment of children with neurodevelopmental disorders (e.g., autism spectrum disorders, ADHD, and learning disorders). The assessment procedure was as follows. During the first encounter, the child was interviewed by the psychiatrist to determine a DSM diagnosis. Then, the psychologist conducted a clinical interview with the child in order to assess additional areas associated with mental health domains. Then, the psychologist conducted an interview with the parents and asked them to complete the CPRS-R, while, the Rorschach was administered to the child according to the R-PAS guidelines by a clinician in training. Usually, the assessment procedure took about three to four hours.

\section{Measures}

\section{Rorschach Performance Assessment System (R-PAS).}

R-PAS includes five interpretative domains: Administration Behaviors \& Observations, Engagement \& Cognitive Processing, Perception \& Thinking Problems, Stress \& Distress and Self \& Other Representation domains. R-PAS raw scores may be converted into easy-to-use, normalized standard scores (SS), which have a 
ADHD AND THE RORSCHACH

mean of 100 and standard deviation of 15 . For the present study, we used the children international norms implemented in R-PAS, so that a score of 100 would indicate the average value of the children's normative group. According to the R-PAS manual, scores may be interpretable and potentially clinically relevant when

Page 1 scores are at or below a standard score of about 90 or when they are at or above a standard score of about 110, and when Page 2 scores are at or below a standard score of about 85 or when they are at or above a standard score of about 115 .

The R-PAS children international norms (Meyer, Viglione, \& Giromini, 2016) were developed to overcome two major problems related to the CS norms for children and adolescents: the discrepancy between official CS norms and research findings and the excessive variability in some scores across different international samples (Meyer et al., 2007). First, the R-PAS authors used two statistical procedures (i.e., continuous norming and bootstrapping) to maximize their ability to distinguish developmental changes in Rorschach protocols; second, they predicted means and standard deviations for each variable from 6 through 17 years of age (for a detailed description of the procedure see Meyer et al. 2016). As a result of this procedure, raw scores are transformed into age-specific standard scores for each variable, indicating how this child or adolescent compares to others of the same age.

Twenty-nine (93.5\% of the sample) Rorschach records were coded twice by two independent raters to assess inter-rater reliability of R-PAS variables. Then, we computed intraclass correlation coefficients (ICCs) for each R-PAS variable. For the present study, the mean ICC was $.80(S D=.17)$ and the median ICC was .87 , ranging from .26 to 1.00. According to the guidelines suggested by Cicchetti (1994) and Shrout and Fliess (1979), 43 variables yielded an excellent reliability (ICC $\geq .75$ ), 11 variables showed good reliability (ICC between .60 and .74), five variables showed fair reliability (ICC between .40 and .59), and only one variable, i.e., the Color Dominance Proportion $[(\mathrm{CF}+\mathrm{C}) / \mathrm{SumC}$; ICC $=.26]$, yielded a poor reliability $(\mathrm{ICC}<.40)$.

\section{Data Analyses}

First, we evaluated the normality of the scores' distributions. Two variables (i.e., T and r) departed substantially from normality (i.e., skewness $<2$ and kurtosis $<7$; West et al. 1995). The reason behind this phenomenon relies on the fact that these two variables have zero-inflated distributions with very low base rates (Kivisalu, et al., 2016; Pignolo et al., 2017): in our sample, 93.5\% of the children provided zero Texture (T) responses, whereas $87.1 \%$ of the children reported zero Reflection (r) responses. Given the lack of variability and the extreme values of skew and kurtosis found for these variables, we excluded these two variables from the analysis. Finally, given that R-PAS proportion scores cannot be computed when the denominator is equal to 
ADHD AND THE RORSCHACH

zero, also MAP/MAHP was excluded from the analysis because it was calculated only for three protocols. Next, for each variable we computed a one-sample t-test, to evaluate whether our sample differed from the R-PAS children's normative group. To control for experiment-wise, Type 1 and Type 2 error for our 57 significance tests, we used stepwise, multistage significance testing, a procedure that allows the preservation of statistical power in exploratory analyses while protecting against inflated alpha (Howell, 2013).

\section{Results}

Table 1 presents the descriptive statistics for the R-PAS variables standard scores with the means of 100 and standard deviations of 15 . Five variables were significantly lower than the corresponding R-PAS children’s normative group variables, with standard scores at or below 90: Human Movement and Weighted Color $\left(\mathrm{MC} ; M=90.2, S D=13.0, t_{(30)}=-4.19\right)$, Human Movement $\left(M ; M=89.1, S D=13.4, t_{(30)}=-4.51\right)$, Form Quality Ordinary percentage $\left(\mathrm{FQo} \% ; M=79.8, S D=11.5, t_{(30)}=-9.81\right)$, Popular $\left(\mathrm{P} ; M=85.0, S D=11.4, t_{(30)}=\right.$ -7.30), and Whole Human Content $\left(\mathrm{H} ; M=88.1, S D=13.3, t_{(30)}=-4.98\right)$. Seven variables were significantly greater than the R-PAS children's normative group, with scores at or above 110 standard scores: Ego Impairment Index-3 (EII-3; $\left.M=114.6, S D=19.1, t_{(30)}=4.27\right)$, Thought \& Perception Composite (TP-Comp; $M$ $\left.=115.2, S D=20.5, t_{(30)}=4.11\right)$, Form Quality Minus percentage $\left(F Q-\% ; M=114.5, S D=19.8, t_{(30)}=4.06\right)$, WD Minus (WD-\%; $\left.M=116.8, S D=18.2, t_{(30)}=5.13\right)$, Suicide Concern Composite $(\mathrm{SC}-\mathrm{Comp} ; M=109.9, S D=$ 11.2, $\left.t_{(30)}=4.93\right)$, Poor Human Responses Proportion (PHR/GPHR; $\left.M=118.8, S D=16.1, t_{(23)}=5.70\right)$, and Form Quality Unusual percentage (FQu\%; $\left.M=112.2, S D=15.5, t_{(30)}=4.36\right)$.

Moreover, we inspected the mean scores of the Rorschach variables that characterized the ADHD profile in previous studies: Color responses [Form-Dominated Color responses (FC; $M=97.3, S D=9.0$ ), ColorDominated with Form secondary responses (CF; $M=97.8, S D=12.4)$, and the Pure C codes $(C ; M=100.7, S D$ = 10.3)]; Animal responses [Whole Animal, A: $M=101.0, S D=18.2$; Whole Animal-Like, (A): $M=106.1, S D$ = 15.5; Animal Detail, Ad: $M=96.2, S D=16.7$; Animal-Like Detail, (Ad): $M=103.6, S D=11.5$ ]; and the number of Common Detail (D, $M=94.9, S D=18.4)$. However, all these variables showed average scores.

\section{Discussion}

The aim of the current study was to evaluate personality characteristics and processing features in children with ADHD in the absence of medication by using R-PAS. From the comparison of R-PAS protocols of 31 children with ADHD with the R-PAS international children's norms, the main findings are related to the presence of reality testing problems (FQo\% and P), unconventional perception (FQu\%), distortions or misinterpretations, and confused or illogical thinking and reasoning leading to poor judgment or unconventional 
ADHD AND THE RORSCHACH

behavior (EII-3, TP-Comp, FQ-\%, and WD-\%). Moreover, the average ADHD profile seems characterized by a problematic or less adaptive understanding of self and others (PHR/GPHR), difficulties mentalizing human behavior (H), lack of ability to elaborate human experience or activities (M), and limited psychological resources (MC). Finally, the Suicide Concern Composite (SC-Comp) could indicate the presence of selfdestructive behaviors and desperation if one generalizes from adult interpretation. However, SC-Comp score was only mildly elevated, and it has not been researched in children.

Our results partially replicated those reported by previous authors. First of all, as reported by Bartell \& Solanto (1995), Cotugno (2005), and Gordon and Oshman (1981), clinical information suggested the presence of reality testing problems and distortion or misinterpretation of external stimuli (low $\mathrm{X}+\%, \mathrm{~F}+\%$, $\mathrm{P}$, and high $\mathrm{X}-\%$ ), and difficulties in understanding and interacting with others (low $\mathrm{M}$ and $\mathrm{H}$ ). Moreover, children with ADHD appeared to have limited psychological resources (low EA), as indicated by Meehan et al. (2008).

Contrary to previous findings, our sample did not show divergent scores from the mean for chromatic color-related variables. The Color Dominance Proportion $[(\mathrm{CF}+\mathrm{C}) / \mathrm{SumC}]$, a measure of cognitive control and modulation in reaction to the environment, and the Weighted Sum of Color (WSumC), which is related to an interest in and awareness of the environment, were both in the average range (Table 1). Looking at the single components of these variables, Form-Dominated Color responses (FC), Color-Dominated with Form secondary responses (CF), and the Pure C codes (C) also showed average scores. Also, the number of responses on cards VIII, IX, and X (eight-nine-ten percent, R8910\%, previously Afr in the Comprehensive System), which refers to a general responsiveness to compelling stimuli, was not clinically relevant (Table 1). Additionally, Gordon and Oshman (1981) reported more Animal contents in hyperactive children, whereas our findings showed an average number of Animal responses (Whole Animal, A; Whole Animal-Like, (A); Animal Detail, Ad; AnimalLike Detail, (Ad)]. Finally, both the Sum of Shading and Achromatic Color (YTVC') and the number of Common Detail (D) were not clinically significant, contrary to the findings by Cotugno (2005) and Jain et al. (2005) respectively.

Together, the limitations highlighted by the R-PAS data might partially explain problems in psychological functioning, coping, and academic performance encountered by children with ADHD. Indeed, children with ADHD are characterized by poor concentration and impulsiveness (Harpin, 2005), lower grades and greater school suspension and grade retention (Barkley, 2002), and poorer perceptions by teachers (Eisenberg \& Schneider, 2007). Both in childhood and adolescence, emotion regulation deficits and impulsiveness induce interpersonal conflict and interfere with relationships and social skills (Bagwell, Molina, 
ADHD AND THE RORSCHACH

Pelham \& Hoza, 2001). Other clinical problems can be related to persistent sadness and hopelessness. Children with ADHD are more likely than children without ADHD to develop childhood depression, and depressive symptoms occurring in childhood can represent a high risk for both single and recurrent episodes of adolescent depression and for suicidal behavior. ADHD in early childhood may predict later depression, and suicidal ideation and suicide attempts (Chronis-Tuscano et al., 2010).

Finally, parent and teacher rating scales of ADHD symptoms in children are only moderately correlated (Achenbach, 2011; De Los Reyes, 2013; Dirks, De Los Reyes, Briggs-Gowan, Cella, \& Wakschlag, 2012). These moderate correlations may be associated to the systematic measurement error due to response styles, rating biases, unique perspectives of raters, and variations in actual behavior and emotions across situations. Despite the sources of disagreement among raters of ADHD, clinicians usually use the combination of parent and teacher rating scales of ADHD symptoms to assess ADHD. Given that a valid diagnosis of ADHD can be crucial for optimal treatment, the Rorschach test may allow clinicians and researchers to examine in depth the individual's personality and implicit processing. In addition, ratings by the various informants are limited to brief descriptions and frequencies of behaviors ensconced in the test items. These contrast with the implicit nature of the Rorschach data expressed in the child's description of what they see in the inkblots. The information is not necessarily filtered through the self-schema nor does not relate to the conscious awareness of the child (see Bornstein, Rossner, Hill, \& Stepanian, 1994). Overall, our results are consistent with those obtained by behavioral studies; for example, these studies reported the presence of misinterpretations and confused thinking, and unconventional behaviors, in line with our results (e.g., Barkley, 1997). However, the findings of the current study furthermore provide information on impairments in specific psychological domains (e.g., Self \& Other Representation, Stress \& Distress) and they highlighted those adaptive and maladaptive personality features (e.g. feelings of desperation, and problematic understanding of self and others and human behavior) characterizing the overall psychological functioning in children with ADHD.

\section{Limitations}

Although our findings provide useful information for understanding the personality functioning (and not exclusively manifest symptoms) of children with ADHD, the study presents some limitations that are worth noting. First, the personality features identified in the current study may have been influenced by environmental factors, such as physical and/or psychological abuse, infection, alcohol and environmental intoxicants, and/or genetic and physiological factors, such as heritability of ADHD, metabolic deficits, and neurological problems (Nikolas \& Burt, 2010). Secondly, we could not evaluate potential gender differences because our sample 
ADHD AND THE RORSCHACH

included only six females. Third, although the using of R-PAS norms for children and adolescents provides a comparison of each Rorschach profile to others of the same age, we compared the Rorschach profiles to norms rather than a matched control group. Indeed, a comparison between our sample and a contrast group would be desirable to investigate and identify those specific characteristics that may distinguish children with ADHD from other clinical groups and non-patients with an opportunity to control demographic and other extraneous variables and to minimize error variance. Fourth, the Color Dominance Proportion $[(\mathrm{CF}+\mathrm{C}) / \mathrm{SumC}]$, which showed contradictory results in the previous studies, yielded a poor inter-rater reliability (ICC $<.40$ ) in the present study. However, inter-rater reliability of the variables that compose the Color Dominance Proportion $[(\mathrm{CF}+\mathrm{C}) /$ SumC $]$ yielded fair to excellent reliability values $(\mathrm{CF}$ : ICC = .44; C: ICC = .52; SumC: ICC = .90). Despite these limitations, the current study has uniquely contributed to the literature by using R-PAS to evaluate the psychological functioning of children with ADHD who were not on medication.

Finally, it is worth noting that the Howell (2013) multistage significance testing, a procedure used in this exploratory study, is quite conservative. It eliminated 18 variables with $p<.05$ from consideration, of which 16 were consistent with other findings in the study or observed correlates of ADHD. They include variables associated with poor interpersonal capacity (Low--COP, MAH, SumH \& High--NPH/SumH), aggressive ideation (High--AGM and AGC), stress and distress (High--C', MOR), limited coping ability and internal resources (MC - PPD, M/MC, p/a+p), confused or illogical thinking (High--WSumCog, SevCog). Trends associated with simplistic processing were inconsistent: High Complexity, F\%, and Low Intellectual Content are associated with simplistic processing whereas high Synthesis and low Vg\% suggest more differentiated and integrated thinking. Given these statistical trends, these variables should be considered for future research.

\section{Author Contribution}

AA: designed and executed the study, assisted with the data analyses, and wrote the paper. CP: analyzed the data and wrote the results. DJV: collaborated with the design and writing of the study. AZ: collaborated with the design and writing of the study. SC: collaborated in the writing and editing of the final manuscript. LF: collaborated with the design and editing of the final manuscript.

\section{Ethical Approval}

For our study, the head physician of the neuropsychiatric ward of the hospital approved the use of archival data from outpatients. All parents gave their informed consent before starting the diagnostic assessment. All 
ADHD AND THE RORSCHACH

procedures performed in studies involving human participants were in accordance with the ethical standards of the institutional and/or national research committee and with the 1964 Helsinki declaration, its later amendments, and comparable ethical standards. 
ADHD AND THE RORSCHACH

\section{References}

Achenbach, T. M. (2011). Commentary: Definitely more than measurement error: But how should we understand and deal with informant discrepancies? Journal of Clinical Child and Adolescent Psychology, 40, 80- 86. doi.org/10.1080/15374416.2011.533416

American Psychiatric Association (2013). Diagnostic and statistical manual of mental disorders (5th ed.). Washington, DC: Author.

Ames, L. B., Metraux, R., Rodell, J. L., \& Walker, R. N. (1974). Child Rorschach responses: Developmental trends from ten to sixteen years. New York, NY: Brunner/Mazel.

Ando’, A., Salatino, A., Giromini, L., Ricci, R., Pignolo, C., Cristofanelli, S., Ferro, L., Viglione, D. J., \& Zennaro, A. (2015). Embodied simulation and ambiguous stimuli: The role of the mirror neuron system. Brain Research, 1629, 135-142. doi: 10.1016/j.brainres.2015.10.025

Bagwell, C.L., Molina, B.S., Pelham, W.E. Jr, \& Hoza, B. (2001). Attention-deficit hyperactivity disorder and problems in peer relations: predictions from childhood to adolescence. Journal of the American Academy of Child and Adolescent Psychiatry, 40, 1285-92. doi: 10.1097/00004583-200111000-00008

Barkley, R. A. (2000). Genetics of childhood disorders: XVII. ADHD, Part 1: The executive functions and ADHD. Journal of the American Academy of Child and Adolescent Psychiatry, 39, 1064-1068. doi: $10.1097 / 00004583-200008000-00025$

Barkley, R. A. (2002). Major life activity and health outcomes associated with attention-deficit/hyperactivity disorder. Journal of Clinical Psychiatry, 63, 10-15.

Barkley, R. A. (2015). Attention-deficit hyperactivity disorder: A handbook for diagnosis and treatment (4th ed.). New York, NY: Guilford Press.

Barkley, R.A. (1997). Behavioral inhibition, sustained attention, and executive functions: constructing a unifying theory of ADHD. Psychological Bulletin, 121, 65-94. doi: 10.1037/0033-2909.121.1.65

Bartell, S. S., \& Solanto, M. V. (1995). Usefulness of the Rorschach inkblot test in assessment of attention deficit hyperactivity disorder. Perceptual and Motor Skills, 80, 531-541. doi:10.2466/pms.1995.80.2.531

Beck, S. J., \& Molish, H. B. (1967). Rorschach’s test. II: A variety of personality pictures. New York, NY: Grune \& Stratton.

Beck, S. J., Beck, A. G., Levitt, E. E., \& Molish, H. B. (1961). Rorschach’s Test: I. Basic Processes. New York, NY: Gurne \& Strattons. 
ADHD AND THE RORSCHACH

Bornstein, R. F., Rossner, S. C., Hill, E. L., \& Stepanian, M. L. (1994). Face validity and fakability of objective and projective measures of dependency. Journal of Personality Assessment, 63, 363-386.

Cassell, W. A. (1980). Body symbolism and the somatic inkblot series. Anchorage, AK: Aurora Publication.

Chronis-Tuscano, A., Molina, B.S. G., Pelham, W.E., Applegate, B., Dahlke, A., Overmyer, M., \& Lahey, B. B. (2010). Very Early Predictors of Adolescent Depression and Suicide Attempts in Children With Attention-Deficit/Hyperactivity Disorder. Archives of General Psychiatry, 67, 1044-1051. doi:10.1001/archgenpsychiatry.2010.127

Cicchetti, D. V. (1994). Guidelines, criteria, and rules of thumb for evaluating normed and standardized assessment instruments in psychology. Psychological Assessment, 6, 284-290. doi: 10.1037/10403590.6.4.284

Conners, C. K., Sitarenios, G., Parker, J. D., \& Epstein, J. N. (1998). The revised Conners’ Parent Rating Scale (CPRS-R): factor structure, reliability, and criterion validity. Journal of Abnormal Child Psychology, 26, 257-268. doi: 10.1023/A:1022602400621

Cotugno, A. J. (1995). Personality attributes of attention deficit hyperactivity disorder (ADHD) using the Rorschach Inkblot Test. Journal of Clinical Psychology, 51, 554-562. doi: 10.1002/10974679(199507)51:4<554::AID-JCLP2270510415>3.0.CO;2-Y

De Los Reyes, A. (2013). Strategic objectives for improving understanding of informant discrepancies in developmental psychopathology research. Development and Psychopathology, 25, 669-882. doi.org/10.1017/S0954579413000096

Dirks, M.A., De Los Reyes, A., Briggs-Gowan, M.J., Cella, D., \& Wakschlag, L.S. (2012). Embracing not erasing contextual variability in children's behavior: Theory and utility in the selection and use of measures and informants in the assessment of developmental psychopathology. Journal of Child Psychology and Psychiatry, 53, 558-574. doi: 10.1111/j.1469-7610.2012.02537.x

Doyle, A. E. (2006) Executive functions in attention-deficit/hyperactivity disorder. Journal of Clinical Psychiatry, 67(suppl 8), 21-26.

DuPaul, G. J., Power, T. J., Anastopoulos, A. D., Reid, R., McGoey, K. E., \& Ikeda, M. J. (1997) Teacher ratings of attention deficit hyperactivity disorder symptoms: Factor structure and normative data. Psychological Assessment, 9, 436-444. doi: 10.1037/1040-3590.9.4.436

Eisenberg, D., \& Schneider, H. (2007). Perceptions of academic skills of children diagnosed with ADHD. Exner, J. E. (1974). The Rorschach: A Comprehensive System: Vol. 1. New York, NY: Wiley. 
ADHD AND THE RORSCHACH

Exner, J. E. (1993). The Rorschach: A comprehensive system: Vol. 1. Basic foundations (3rd ed.). New York, NY: Wiley.

Exner, J. E. (2003). The Rorschach: a comprehensive system. Vol. 1: Basic Foundations and Principles of Interpretation (4th ed). Hoboken, NJ: Wiley.

Gershon, J (2002). A meta-analytic review of gender differences in ADHD. Journal of attention disorders 5, 143-54. doi:10.1177/108705470200500302

Giromini, L., Ando’, A., Morese, R., Salatino, A., Di Girolamo, M., Viglione, D. J., \& Zennaro, A. (2016). Rorschach Performance Assessment System (R-PAS) and vulnerability to stress: A preliminary study on electrodermal activity during stress. Psychiatry Research, 246, 166-172. doi: 10.1016/j.psychres.2016.09.036

Giromini, L., Viglione, D. J., \& McCullaugh, J. (2015). Introducing a Bayesian approach to determining degree of fit with existing Rorschach norms. Journal of Personality Assessment, 97, 354-363. doi: 10.1080/00223891.2014.959127

Giromini, L., Viglione, D. J., Zennaro, A., \& Cauda, F. (2017). Neural activity during production of Rorschach responses: An fMRI study. Psychiatry Research: Neuroimaging, 262, 25-31. doi: 10.1016/j.pscychresns.2017.02.001

Gordon, M., \& Oshman, H. (1981). Rorschach indices of children classified as hyperactive. Perceptual and Motor Skills, 52, 703-707. doi: 10.2466/pms.1981.52.3.703

Graceffo, R. A., Mihura, J. L., \& Meyer, G. J. (2014). A Meta-Analysis of an Implicit Measure of Personality Functioning: The Mutuality of Autonomy Scale. Journal of Personality Assessment, 96, 581-595. doi: $10.1080 / 00223891.2014 .919299$

Hamel, M., \& Shaffer, T. W. (2007). Rorschach Comprehensive System data for 100 nonpatient children from the United States in two age groups. Journal of Personality Assessment, 89, S174-S182. doi: $10.1080 / 00223890701583457$

Harpin, V.A. (2005). The effect of ADHD on the life of an individual, their family, and community from preschool to adult life. Archives of Disease in Childhood, 90, 2-7. doi: 10.1136/adc.2004.059006

Howell, D. C. (2013). Statistical methods for psychology (8th ed.). Belmont, CA: Wadsworth Cengage Learning. 
ADHD AND THE RORSCHACH

Jain, J., Singh, B., Mohanty, S., \& Kumar, R. (2005). SIS-I and Rorschach Diagnostic Indicators of Attention

Deficit and Hyperactivity Disorder. SIS Journal of Projective Psychology \& Mental Health, 12, 141152.

Journal of Attention Disorders, 10, 390-7. doi: 10.1177/1087054706292105

Kivisalu, T. M., Lewey, J. H., Shaffer, T. W., \& Canfield, M. L. (2016). An investigation of interrater reliability for the Rorschach Performance Assessment System (R-PAS) in a nonpatient U.S. sample. Journal of Personality Assessment, 98, 382-390. doi: 10.1080/00223891.2015.1118380

Klopfer, B., \& Kelley, D. M. (1942). The Rorschach technique: A manual for a projective method of personality diagnosis. Yonkers on Hudson, NY: Word Books.

Klopfer, B., Ainsworth, M. D., Klopfer, W. G., \& Holt, R. R. (1954). Developments in the Rorschach Technique. Vol. I. Techniques and Theory. Yonkers on Hudson, NY: World Books.

Lewey, J. H., Kivisalu, T. M., \& Giromini, L. (2018). Coding With R-PAS: Does Prior Training With the Exner Comprehensive System Impact Interrater Reliability Compared to Those Examiners With Only R-PASBased Training? Journal of Personality Assessment. doi: 10.1080/00223891.2018.1476361

Martel, M. M., Schimmack, U., Nikolas, M., \& Nigg, J. T. (2015). Integration of symptom ratings from multiple informants in ADHD diagnosis: a psychometric model with clinical utility. Psychological Assessment, 27, 1060-71. doi: 10.1037/pas0000088.

Meehan, K. B., Ueng-McHale, J. Y., Reynoso, J. S., Harris, B. H., Wolfson, V. M., Gomes, H., \& Tuber, S. B. (2008). Self-regulation and internal resources in school-age children with ADHD symptomatology: An investigation using the Rorschach inkblot method. Bulletin of the Menninger Clinic, 72, 259-282. doi: 10.1521/bumc.2008.72.4.259

Meyer, G. J. (2017). What Rorschach performance can add to assessing and understanding personality. International Journal of Personality Psychology, 3(1), 36-49.

Meyer, G. J., Erdberg, P., \& Shaffer, T. W. (2007). Towards international normative reference data for the Comprehensive System. Journal of Personality Assessment, 89, S201-S216. doi: $10.1080 / 00223890701629342$

Meyer, G. J., Shaffer, T. W., Erdberg, P., \& Horn, S. L. (2015) Addressing issues in the development and use of the Composite International Reference Values as Rorschach norms for adults. Journal of Personality Assessment, 97, 330-347. doi: 10.1080/00223891.2014.961603 
ADHD AND THE RORSCHACH

Meyer, G. J., Viglione, D. J., \& Exner, J. E. (2001). Superiority of Form\% over Lambda for research on the Rorschach Comprehensive System. Journal of Personality Assessment, 76, 68-75. doi: 10.1207/S15327752JPA7601_4

Meyer, G. J., Viglione, D. J., \& Giromini, L. (2016). Current R-PAS Transitional Child and Adolescent Norms. Retrieved from http://r-pas.org/CurrentChildNorms.aspx

Meyer, G. J., Viglione, D. J., Mihura, J. L., Erard, R. E., \& Erdberg, P. (2011). A manual for the Rorschach Performance Assessment System. Toledo, OH: R-PAS.

Mihura, J. L., Meyer, G. J., Dumitrascu, N., \& Bombel, G. (2013). The validity of individual Rorschach variables: Systematic reviews and meta-analyses of the Comprehensive System. Psychological Bulletin, 139, 548-605. doi: 10.1037/a0029406

Nikolas, M. A., \& Burt, S.A. (2010). Genetic and Environmental Influences on ADHD Symptom Dimensions of Inattention and Hyperactivity: A Meta-Analysis. Journal of Abnormal Psychology, 119, 1-17. doi: 10.1037/a0018010

Pignolo, C., Giromini, L., Ando’, A., Ghirardello, D., Di Girolamo, M., Ales, F., \& Zennaro, A. (2017). An interrater reliability study of Rorschach Performance Assessment System (R-PAS) raw and complexity-adjusted scores. Journal of Personality Assessment, 99, 619-625. doi: 10.1080/00223891.2017.1296844

Rapaport, D., Gill, M. M., Schafer, R., \& Holt, R. R. (1968). Diagnostic psychological testing (Rev. ed.). New York, NY: International Universities Press.

Reese, J. B., Viglione, D. J., \& Giromini, L. (2014). A comparison between Comprehensive System and an early version of the Rorschach Performance Assessment System administration with outpatient children and adolescents. Journal of Personality Assessment, 96, 515-522. doi: 10.1080/00223891.2014.889700

Reynolds, C. R., \& Kamphaus, R. W. (1992). Behaviour Assessment System for Children Manual. Circle Pines, MN: American Guidance Service.

Ross, D. M., \& Ross, S. A. (1976). Hyperactivity: Research, theory, and action. New York, NY: Wiley.

Shrout, P. E., \& Fleiss, J. L. (1979). Intraclass correlations: Uses in assessing rater reliability. Psychological Bulletin, 86, 420-428. doi: 10.1037/0033-2909.86.2.420

Vaidya, C. J. (2012). Neurodevelopmental abnormalities in ADHD. Current Topics in Behavioral Neurosciences, 9, 49-66. doi: 10.1007/7854_2011_138 
ADHD AND THE RORSCHACH

Viglione, D. J. (1999). A review of recent research addressing the utility of the Rorschach. Psychological Assessment, 11, 251-265. doi: 10.1037/1040-3590.11.3.251

Viglione, D. J., \& Giromini, L. (2016). The effects of using the International versus Comprehensive System norms for children, adolescents, and adults. Journal of Personality Assessment, 98, 391-397. doi: $10.1080 / 00223891.2015 .1136313$

Viglione, D. J., \& Hilsenroth, M. J. (2001). The Rorschach: Facts, fictions, and future. Psychological Assessment, 13, 452-471. doi: 10.1037//1040-3590.13.4.452

Viglione, D. J., Blume-Marcovici, A. C., Miller, H. L., Giromini, L., \& Meyer, G. J. (2012). An inter-rater reliability study for the Rorschach performance Assessment System. Journal of Personality Assessment, 94, 607-612. doi: 10.1080/00223891.2012.684118

Viglione, D. J., Giromini, L., Gustafson, M., \& Meyer, G. J. (2014). Developing continuous variable composites for Rorschach measures of thought problems, vigilance, and suicide risk. Assessment, 21, 42-49. doi: $10.1177 / 1073191112446963$

Weiner, I. B. (1998). Rorschach differentiation pf schizophrenia and affective disorder In G. P. Koocher, J. C. Norcross, \& S. S. Hill (Eds.), Psychologist’s desk reference (pp 151-154). New York, NY: Oxford University Press.

West, S. G., Finch, J. F., \& Curran, P. J. (1995). Structural equation models with non-normal variables: Problems and remedies. In R. Hoyle (Ed.), Structural equation modeling: Concepts, issues, and applications (pp. $56-75)$. Thousand Oaks, CA: Sage.

Wilens, T. E., Biederman, J., \& Spencer, T. J. (2002). Attention Deficit/Hyperactivity Disorder Across the Lifespan. Annual Review of Medicine, 53, 113-131. doi.org/10.1146/annurev.med.53.082901.103945

Wood, J. M., Nezworski, M. T., \& Stejskal, W. J. (1996). The Comprehensive System for the Rorschach: A critical examination. Psychological Science, 7, 3-10. doi: 10.1111/j.1467-9280.1996.tb00658.x

Zhong, S., Jing, J., Wang, L., \& Yin, Q. (2007). Analysis on Rorschach Inkblot Test in Children with Attention Deficit Hyperactivity Disorder. Chinese Journal of Clinical Psychology, 15, 545-547. 


\section{ADHD AND THE RORSCHACH}

Table 1

Descriptive statistics of R-PAS raw standard scores on Page 1 and Page 2 and differences between our sample and the R-PAS children normative sample (one-sample t-test)

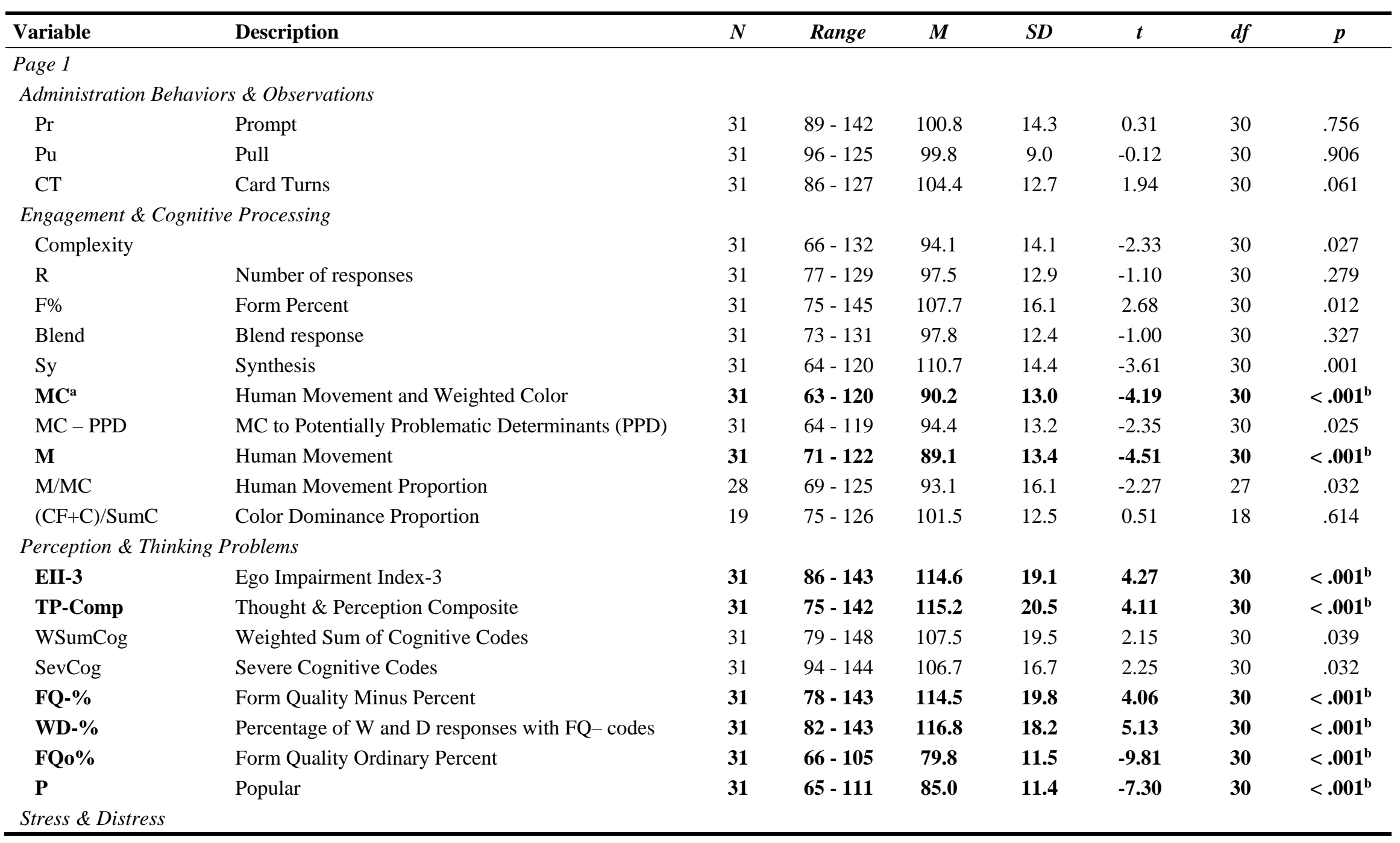


ADHD AND THE RORSCHACH

\begin{tabular}{|c|c|c|c|c|c|c|c|c|}
\hline Variable & Description & $N$ & Range & $M$ & $S D$ & $t$ & $d f$ & $p$ \\
\hline YTVC' & Sum of Shading and Achromatic Color & 31 & $73-139$ & 100.2 & 15.3 & 0.08 & 30 & .935 \\
\hline $\mathrm{m}$ & Inanimate Movement & 31 & $84-137$ & 102.5 & 13.8 & 1.00 & 30 & .325 \\
\hline $\mathrm{Y}$ & Diffuse Shading & 31 & $85-137$ & 98.1 & 13.2 & -0.80 & 30 & .428 \\
\hline MOR & Morbid Content & 31 & $86-143$ & 108.3 & 15.4 & 3.00 & 30 & .005 \\
\hline SC-Comp & Suicide Concern Composite & 31 & $83-128$ & 109.9 & 11.2 & 4.93 & 30 & $<.001^{\mathrm{b}}$ \\
\hline \multicolumn{9}{|c|}{ Self \& Other Representation } \\
\hline SR & Space Reversal & 31 & $87-132$ & 100.0 & 13.1 & -0.01 & 30 & .989 \\
\hline MAP/MAHP & Mutuality of Autonomy Pathology Proportion & 3 & $72-123$ & 95.7 & 25.7 & - & - & - \\
\hline PHR/GPHR & Poor Human Representation Proportion & 24 & $75-136$ & 118.8 & 16.1 & 5.70 & 23 & $<.001^{\mathrm{b}}$ \\
\hline M- & Human Movement determinant with FQ- & 31 & $95-143$ & 103.8 & 12.6 & 1.67 & 30 & .106 \\
\hline AGC & Aggressive Content & 31 & $74-143$ & 108.5 & 18.4 & 2.57 & 30 & .015 \\
\hline $\mathbf{H}$ & Human Content & 31 & $75-113$ & 88.1 & 13.3 & -4.98 & 30 & $<.001^{\mathrm{b}}$ \\
\hline COP & Cooperative Movement & 31 & $88-120$ & 94.0 & 9.5 & -3.55 & 30 & .001 \\
\hline \multicolumn{9}{|l|}{ Page 2} \\
\hline \multicolumn{9}{|c|}{ Engagement \& Cognitive Processing } \\
\hline W\% & Whole Location Response Percent & 31 & $77-132$ & 103.8 & 16.3 & 1.31 & 30 & .201 \\
\hline Dd\% & Unusual Detail Percent & 31 & $75-132$ & 100.0 & 14.3 & 0.01 & 30 & .990 \\
\hline SI & Space Integration & 31 & $74-132$ & 101.6 & 15.9 & 0.55 & 30 & .584 \\
\hline IntCont & Intellectualized Content & 31 & $81-128$ & 91.2 & 14.1 & -3.47 & 30 & .002 \\
\hline Vg\% & Vagueness Percent & 31 & $86-118$ & 95.3 & 10.9 & -2.40 & 30 & .023 \\
\hline FD & Form Dimension & 31 & $88-122$ & 100.9 & 12.3 & 0.42 & 30 & .675 \\
\hline R8910\% & Percentage of Responses on Cards VIII, IX, and X & 31 & $71-129$ & 97.4 & 14.8 & -0.97 & 30 & .340 \\
\hline WSumC & Weigheted Sum of Color & 31 & $70-126$ & 96.1 & 12.9 & -1.67 & 30 & .105 \\
\hline $\mathrm{C}$ & Pure Color & 31 & $95-130$ & 100.7 & 10.3 & 0.34 & 30 & .692 \\
\hline $\mathrm{Mp} /(\mathrm{Ma}+\mathrm{Mp})$ & Passive Human Movement Proportion & 11 & $75-130$ & 100.8 & 16.2 & 0.17 & 10 & .870 \\
\hline \multicolumn{9}{|c|}{ Perception \& Thinking Problems } \\
\hline FQu\% & Form Quality unusual Percent & 31 & $81-143$ & 112.2 & 15.5 & 4.36 & 30 & $<.001^{\mathrm{b}}$ \\
\hline
\end{tabular}


ADHD AND THE RORSCHACH

\begin{tabular}{|c|c|c|c|c|c|c|c|c|}
\hline Variable & Description & $N$ & Range & $M$ & $S D$ & $t$ & $d f$ & $p$ \\
\hline PPD & Potentially Problematic Determinants & 31 & $73-138$ & 98.1 & 16.1 & -0.67 & 30 & .510 \\
\hline CBlend & Color Blended with Shading and Achromatic Color & 31 & $91-117$ & 101.6 & 9.7 & 0.91 & 30 & .371 \\
\hline$C^{\prime}$ & Achromatic Color & 31 & $84-145$ & 108.3 & 14.2 & 3.25 & 30 & .003 \\
\hline $\mathrm{V}$ & Vista & 31 & $92-134$ & 99.3 & 11.6 & -0.36 & 30 & .725 \\
\hline CritCont\% & Critical Contents Percent & 31 & $70-148$ & 105.1 & 18.8 & 1.50 & 30 & .145 \\
\hline \multicolumn{9}{|c|}{ Self \& Other Representation } \\
\hline SumH & All Human Content & 31 & $63-113$ & 93.1 & 14.4 & -2.65 & 30 & .013 \\
\hline NPH/SumH & Non-Pure H Proportion & 25 & $65-127$ & 109.5 & 17.2 & 2.77 & 24 & .011 \\
\hline V-Comp & Vigilance Composite & 31 & $75-131$ & 100.6 & 13.0 & 0.25 & 30 & .806 \\
\hline $\mathrm{r}$ & Reflections & 31 & $95-128$ & 98.1 & 8.5 & - & - & - \\
\hline $\mathrm{p} /(\mathrm{a}+\mathrm{p})$ & Passive Proportion & 28 & $70-137$ & 107.6 & 18.4 & 2.20 & 27 & .036 \\
\hline AGM & Aggressive Movement & 31 & $93-146$ & 107.3 & 17.6 & 2.31 & 30 & .028 \\
\hline $\mathrm{T}$ & Texture & 31 & $91-129$ & 92.7 & 7.3 & - & - & - \\
\hline PER & Personal Knowledge Justification & 31 & $92-131$ & 103.9 & 13.0 & 1.66 & 30 & .108 \\
\hline An & Anatomy & 31 & $85-133$ & 101.5 & 14.1 & 0.59 & 30 & .562 \\
\hline
\end{tabular}

Note. Degree of freedom $(d f)$ differed for R-PAS proportion scores because they may not be computed when the denominator is equal to zero. ${ }^{\text {a }}$ In the CS this variable is

called EA. ${ }^{\text {b }}$ Coefficients are statistically significant following multistage significance testing. 